\title{
Transforming Research and Clinical Knowledge in Spinal Cord Injury (TRACK-SCI): an overview of initial enrollment and demographics
}

\author{
${ }^{*}$ Rachel E. Tsolinas, BA, ${ }^{1}$ John F. Burke, MD, PhD, ${ }^{1,2}$ Anthony M. DiGiorgio, DO, MHA, ${ }^{1,2}$ \\ Leigh H. Thomas, BA, ${ }^{1-3}$ Xuan Duong-Fernandez, BA, ${ }^{1-3}$ Mark H. Harris, BA, ${ }^{1-3}$ John K. Yue, MD, $, 1,2$ \\ Ethan A. Winkler, MD, PhD, ${ }^{1,2}$ Catherine G. Suen, BA, ${ }^{1,2}$ Lisa U. Pascual, MD, ${ }^{4,5}$ \\ Adam R. Ferguson, PhD, ${ }^{1-3,6}$ J. Russell Huie, PhD, ${ }^{1-3}$ Jonathan Z. Pan, MD, PhD, ${ }^{1,7}$ \\ Debra D. Hemmerle, RN, ${ }^{1-3}$ Vineeta Singh, MD, ${ }^{1,3,8}$ Abel Torres-Espin, PhD, ${ }^{1,2}$ \\ Cleopa Omondi, MS,,$^{1-3}$ Nikos Kyritsis, PhD, ${ }^{1-3}$ Jenny Haefeli, PhD, ${ }^{1,2}$ Philip R. Weinstein, MD, ${ }^{2,3,9}$ \\ Carlos A. de Almeida Neto, MA, ${ }^{1-3}$ Yu-Hung Kuo, MD, PhD, ${ }^{12}$ Derek Taggard, MD,12 \\ Jason F. Talbott, MD, PhD, ${ }^{1,10}$ William D. Whetstone, MD,11 Geoffrey T. Manley, MD, PhD, ${ }^{1,2}$ \\ Jacqueline C. Bresnahan, PhD, ${ }^{1-3}$ Michael S. Beattie, PhD, ${ }^{1-3}$ and Sanjay S. Dhall, MD ${ }^{1,2}$ \\ ${ }^{1}$ Brain and Spinal Injury Center, Zuckerberg San Francisco General Hospital; Departments of ${ }^{2}$ Neurological Surgery, ${ }^{5}$ Orthopedic \\ Surgery, ${ }^{7}$ Anesthesia and Perioperative Care, ${ }^{8}$ Neurology, and ${ }^{11}$ Emergency Medicine, University of California San Francisco; \\ ${ }^{3}$ Weill Institutes for Neuroscience; ${ }^{4}$ Orthopaedic Surgery and Orthopedic Trauma Institute, Zuckerberg San Francisco General \\ Hospital; ${ }^{6}$ San Francisco Veterans Affairs Healthcare System; ${ }^{9}$ Institute for Neurodegenerative Diseases, Spine Center, University \\ of California San Francisco; ${ }^{10}$ Department of Radiology and Biomedical Imaging, Zuckerberg San Francisco General Hospital, San \\ Francisco; and ${ }^{12}$ Department of Neurological Surgery, University of California San Francisco-Fresno, Fresno, California
}

OBJECTIVE Traumatic spinal cord injury (SCI) is a dreaded condition that can lead to paralysis and severe disability. With few treatment options available for patients who have suffered from SCl, it is important to develop prospective databases to standardize data collection in order to develop new therapeutic approaches and guidelines. Here, the authors present an overview of their multicenter, prospective, observational patient registry, Transforming Research and Clinical Knowledge in SCl (TRACK-SCI).

METHODS Data were collected using the National Institute of Neurological Disorders and Stroke (NINDS) common data elements (CDEs). Highly granular clinical information, in addition to standardized imaging, biospecimen, and followup data, were included in the registry. Surgical approaches were determined by the surgeon treating each patient; however, they were carefully documented and compared within and across study sites. Follow-up visits were scheduled for 6 and 12 months after injury.

RESULTS One hundred sixty patients were enrolled in the TRACK-SCI study. In this overview, basic clinical, imaging, neurological severity, and follow-up data on these patients are presented. Overall, $78.8 \%$ of the patients were determined to be surgical candidates and underwent spinal decompression and/or stabilization. Follow-up rates to date at 6 and 12 months are $45 \%$ and $36.3 \%$, respectively. Overall resources required for clinical research coordination are also discussed. CONCLUSIONS The authors established the feasibility of SCI CDE implementation in a multicenter, prospective observational study. Through the application of standardized SCI CDEs and expansion of future multicenter collaborations, they hope to advance $\mathrm{SCl}$ research and improve treatment.

https://thejns.org/doi/abs/10.3171/2020.2.FOCUS191030

KEYWORDS SCl; spine trauma; data registries

\footnotetext{
ABBREVIATIONS AIS = ASIA Impairment Scale; ASIA = American Spinal Injury Association; BASIC = Brain and Spinal Injury Center; CDE = common data element; CMS = Centers for Medicare \& Medicaid Services; CRC = clinical research coordinator; GCS = Glasgow Coma Scale; IRB = institutional review board; ISCI = International Spinal Cord Injury; ISCoS = International Spinal Cord Society; ISNCSCI = International Standard for Neurological Classification of Spinal Cord Injury; ISS = Injury Severity Score; LAR = legally authorized representative; NINDS = National Institute of Neurological Disorders and Stroke; REDCap = Research Electronic Data Capture; SCI = spinal cord injury; TBI = traumatic brain injury; TRACK-SCI = Transforming Research and Clinical Knowledge in SCI; UCSF = University of California San Francisco; ZSFGH = Zuckerberg San Francisco General Hospital.
}

SUBMITTED December 31, 2019. ACCEPTED February 14, 2020.

INCLUDE WHEN CITING DOI: 10.3171/2020.2.FOCUS191030.

${ }^{*}$ R.E.T. and J.F.B. share first authorship of this work. 
$\mathrm{T}$ RAUMATIC spinal cord injury (SCI) is a dreaded condition; patients with SCI may live with paralysis, compromised respiratory and cardiovascular systems, sensory impairment, alterations in sexual functioning, loss of bladder and/or bowel control, and severe reflex spasms. ${ }^{22}$ Despite the physical, emotional, and financial cost of SCI, there are few large-scale research directives in this field. However, SCI affects 17,000 people per year in the United States, ${ }^{22}$ which is more common than glioblastoma (approximately 12,000), ${ }^{25}$ operative meningiomas (approximately 15,000), ${ }^{1}$ ruptured arteriovenous malformations (approximately 3000), ${ }^{18}$ and spinal cord tumors (2700). ${ }^{17}$ In addition, SCI patients often live many years after injury, resulting in a relatively high estimated prevalence of 243,000 to 347,000 patients in the United States. ${ }^{22}$ Indeed, this prevalence, coupled with the large financial burden (estimated lifetime direct cost of SCI per patient ranges from $\$ 1.1$ to $\$ 4.7$ million), results in a total societal cost of between $\$ 267$ and $\$ 1631$ billion. ${ }^{9}$ Simply put, SCI is one of the most common, incapacitating, and costly neurological conditions.

Despite its prevalence, there are only a few multicenter prospective databases or clinical trials dedicated to SCI. ${ }^{7,13,24}$ This stands in contrast to other neurosurgical diseases (e.g., glioblastoma and ruptured aneurysms) for which large-scale prospective studies are standard. ${ }^{20,21}$ In particular, there is a lack of documentation of acute injury treatment and outcomes. The dearth of prospective databases leads to underpowered clinical trials, the persistence of institutional biases in treatment algorithms, disjointed data collection efforts, and suboptimal translational research opportunities. ${ }^{30}$ Multicenter databases can help ameliorate these shortcomings by providing an ample, heterogeneous population in which multiple institutions and countries can compare SCI demographics, etiologies, trauma characteristics, treatments, and outcomes. ${ }^{4}$ Furthermore, the use of SCI registries can increase prevention education programs, quality improvement initiatives, and multicentered treatment studies through reducing the time spent on data collection and establishing institutional oversight.

Recognizing the need for more well-funded prospective data registries for SCI, the International Spinal Cord Society (ISCoS) and American Spinal Injury Association (ASIA) led a global effort in 2002 to develop standard SCI variables, ${ }^{23}$ leading to the creation of the International SCI Data Set. ${ }^{3}$ In 2006 the National Institute of Neurological Disorders and Stroke (NINDS) began to develop the Common Data Elements (CDEs) project. ${ }^{2}$ These projects provided guidelines and data collection forms that facilitated systematic data collection and analysis techniques in neurological disorders for the enrichment of data sharing within the neuroscience research community.

With this work as a foundation, the multicenter, prospective, observational patient registry Transforming Research and Clinical Knowledge in SCI (TRACK-SCI) was created in 2013, funded by the Department of Defense (DOD). The aim of this project is to use SCI CDEs to establish a comprehensive data repository infrastructure that houses SCI patient demographics, blood biomarkers, neuroradiological characteristics, and clinical intervention results, as well as neurological, psychological, and functional outcomes at multiple time points across multiple centers following the initial injury. In this paper, we provide an overview of the TRACK-SCI study design and early patient demographic results in order to provide recommendations for future research.

\section{Methods \\ Centers Involved}

TRACK-SCI is a multicenter, prospective observational study conducted by the University of California San Francisco (UCSF) Brain and Spinal Injury Center (BASIC). This study initially recruited patients solely at the Zuckerberg San Francisco General Hospital (ZSFGH), a level I trauma center. Institutional review board (IRB) approval was obtained at this site for all study procedures. The study has since expanded to the UCSF-Fresno hospital and is in the process of bringing on The Ohio State University Medical Center.

\section{Participant Screening, Inclusion, and Exclusion SCI Patients}

All English- and non-English-speaking patients who presented to the emergency department and were diagnosed with a traumatic SCI were potentially eligible for the study. Research staff were notified of a potential participant by neurosurgery clinical staff and daily trauma registry screening conducted by study coordinators, along with other trauma research personnel.

Patients with an age $<18$ years or who were in custody, prisoners, pregnant, or on medically indicated psychiatric hold were excluded. Informed consent was sought for all patients when appropriate. For patients who were unable to sign for themselves because of their injury, a witness unaffiliated with the study was present throughout the consenting process and signed on the patient's behalf. Patients incapable of consenting themselves were initially enrolled via a legally authorized representative (LAR; next of kin) and later approached for patient consent if appropriate. Patients and LARs had the option to participate in all or some of the following study portions: blood draws, International Standard for Neurological Classification of Spinal Cord Injury (ISNCSCI) examinations, and/ or follow-up assessments. Patients were compensated $\$ 50$ after each time point (hospital stay, 3-month phone call, 6-month in-person visit, 12-month in-person visit) for a maximum total of $\$ 200$. Checks or gift cards were dispersed via mail or in person after each time point.

\section{Non-SCI Trauma Control Participants}

In June 2016, non-SCI participant recruitment ("healthy controls") began strictly for the biospecimen and biomarker portion of this study to be used as controls. IRBapproved recruitment flyers were posted around ZSFGH. Non-SCI individuals contacted study coordinators and consented to participate in the study. For these healthy controls, we collected basic demographic information, as well as biospecimens for biomarker research. There was no monetary compensation for participation for healthy controls. In addition, we also collected data on "trauma controls," which were patients with traumatic nonspinal 


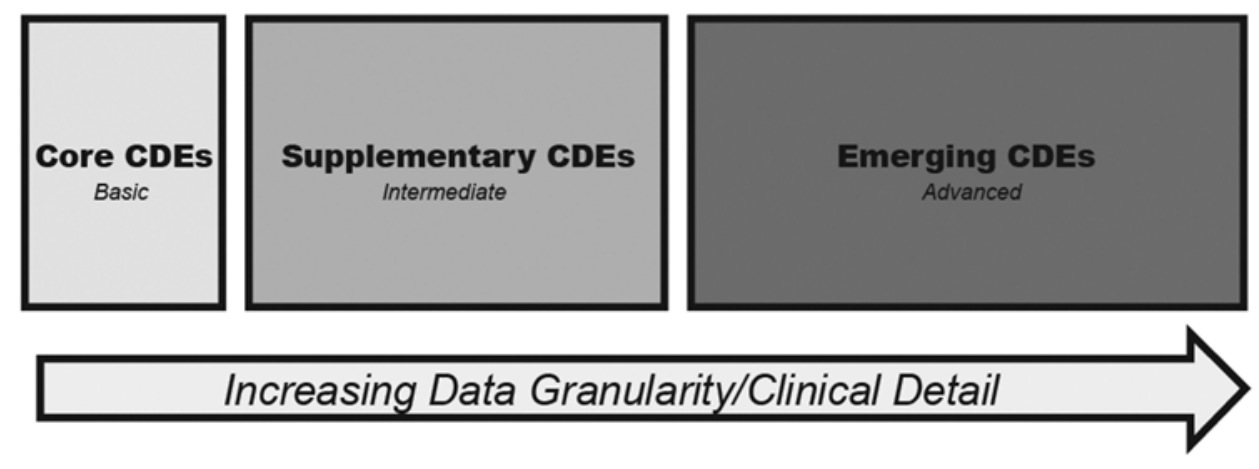

FIG. 1. Classification of CDEs, version 1.0.

injuries. We collected the same CDEs (except for the imaging protocols and surgical data) on these patients for comparison purposes.

\section{Data Collection and Development of the TRACK-SCI Database}

The TRACK-SCI study was modeled after the observational TRACK-TBI (traumatic brain injury) study. ${ }^{34}$ As in TRACK-TBI, the NINDS CDEs were the foundation of our TRACK-SCI database. Core CDEs are data elements that all SCI studies are strongly encouraged to use in the collection of basic participant information. Supplemental CDEs are recommended for SCI clinical research but vary in relevance (e.g., study design, type of intervention). ${ }^{2}$ The supplemental CDEs that are highly relevant to specific types of SCI studies (e.g., clinical trial, cohort study, acute or chronic, phase $1 / 2$ or 3 , etc.) are subcategorized as "supplemental/highly recommended." ${ }^{3}$ Emerging CDEs require further validation before consensus can be achieved (Fig. 1).

Additional measures from the ISCoS were used. Data collection domains are listed in Table 1 and include demographic, clinical, radiological, and functional outcome measures. All data collected for these CDEs were housed in a Research Electronic Data Capture (REDCap) database. The REDCap database was designed from May 2015 to August 2015. The process involved reviewing CDEs, assembling the data dictionary, and setting up the database. More than 21,000 data fields were created when considering the additional institutional variables, calculated fields, repeated measures, date and time stamping of measures, and completion status log. From August 2015 to November 2015 , the REDCap database underwent functionality testing and was ready for use in December 2015. REDCap is in full compliance with Health Insurance Portability and Accountability Act (HIPAA) security standards for the protection of personal health information.

\section{Demographic and Clinical Data}

The following CDE categories composed the demographic and clinical data domain: 1) demographics, 2) health history, 3) injury-related events, and 4) assessments and examinations. Table 1 provides further detail on the specific CDEs used. A total of 229 variables concerning patient demographics, medical history, and consent and contact information were collected through abstraction from electronic medical record systems and patient interviews. Upon admission to the inpatient service, another 19,148 variables regarding trauma characteristics, injury severity, blood pressure management, operating room procedures, interventions, hospital outcomes, high-frequency operating room vital signs, and motor and sensory examinations and pain questionnaires were obtained from both paper and electronic medical records.

\section{Motor and Sensory Examinations}

The ISNCSCI is published by the ASIA and was used to assess motor function and sensory impairment and group patients by injury severity. ${ }^{19}$ Specifically, this examination provides a measure of SCI severity based on the ASIA Impairment Scale (AIS; see Kirshblum et al. ${ }^{19}$ for a review) and ranges from $\mathrm{A}$ (most severe) to $\mathrm{E}$ (not impaired). ISNCSCI examinations were conducted by physicians, nurse practitioners, physician assistants, and ICU nurses who had completed the ASIA International Standards Training e-Learning Program (InSTeP) and in-person training. ISNCSCI examinations were performed during the initial admission for all patients, either as part of clinical care if the treating provider had completed InSTeP training or separately for the research study if the ISNCSCI was not performed for clinical purposes. Occasionally, an ISNCSCI assessment was not performed during the admission, usually because the patient was excessively sedated and could not participate. If a reliable ISNCSCI examination was possible, patients completed examinations at regular intervals including admission (day $0=0-23$ hours from injury), every 48 hours until postinjury day 7 , discharge, 6 -month follow-up ( \pm 2 weeks), and 12-month follow-up ( \pm 2 weeks).

\section{Biospecimens and Biomarkers: SCI Patient Samples}

From May 2015 to August 2015, only blood serum was collected within 24 and 48 hours of injury. From September 2015 to April 2016, additional blood serum samples were collected at the 6- and 12-month follow-ups as well as the inpatient time points. Starting May 2016, serum samples were collected within $24,48,72$, and 120 hours postinjury and at the 6- and 12-month follow-up assessments. Blood plasma collection for white blood cells or for RNA transcriptomics began in April 2016 for the assess- 
TABLE 1. Comprehensive list of measures and CDEs collected in TRACK-SCI

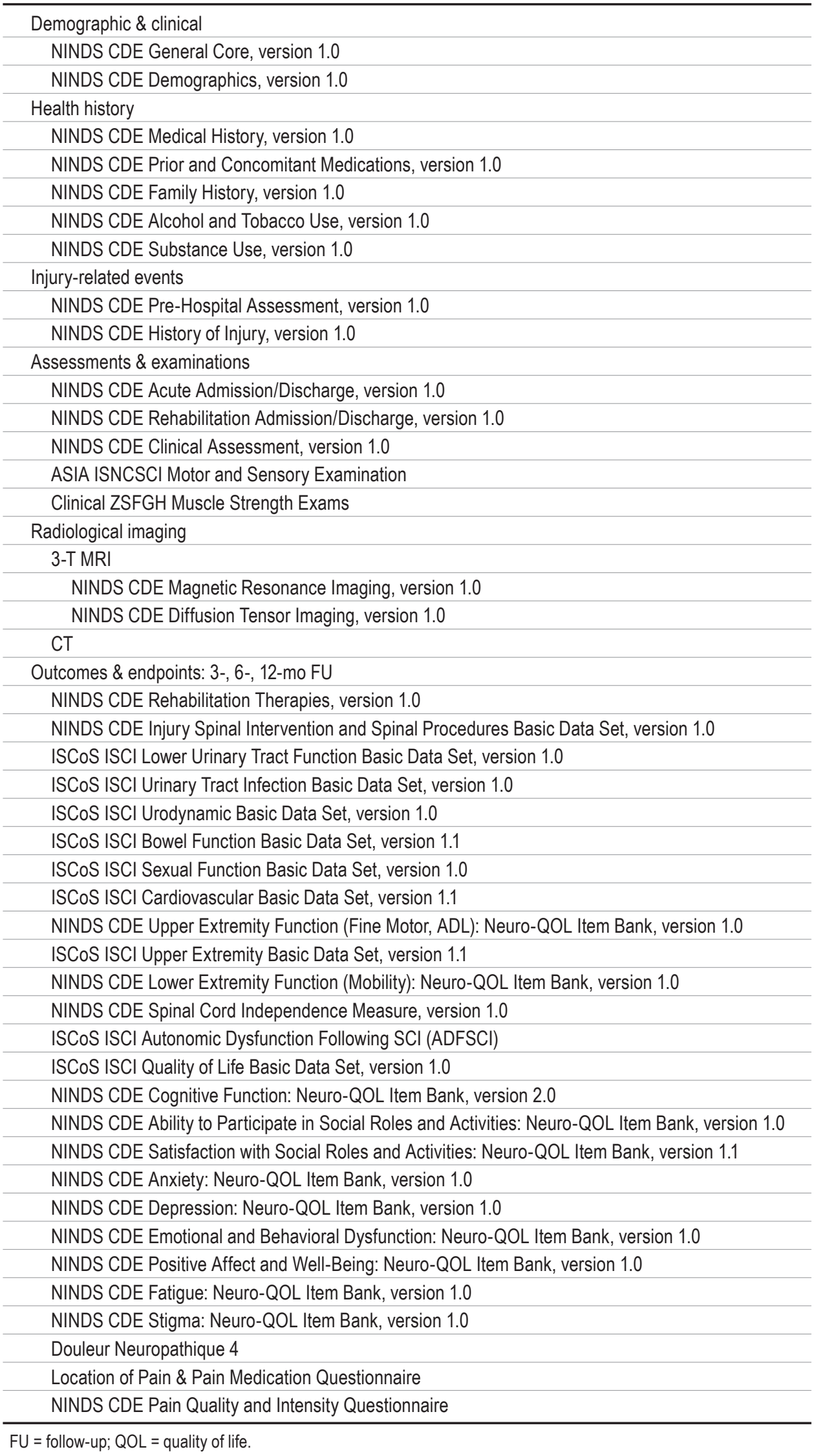


ments 24 , 48, and 72 hours after injury as well as the 6and 12-month assessments. All blood biospecimen collection and processing were conducted by trained personnel.

In lieu of CDE working group guidelines for SCI proteomic preservation of plasma and isolation of DNA, TRACK-TBI protocols were used. TRACK-TBI's processing and storage parameters were based on the CDE working group guidelines for TBI proteomic and genomic preservation and isolation. At each time point the samples were aliquoted and frozen at $-80^{\circ} \mathrm{C}$ within 1 hour of collection. ${ }^{34}$ Blood was collected in 6-ml Vacuette $\mathrm{Z}$ serum clot activator tubes for serum extraction. To prevent reduction in sample volume, externally threaded cryovials were used for serum storage. The serum was divided into multiple 500- $\mu$ l aliquots for storage. An inventory system was developed to track time of collection, processing, and storage for all biospecimens.

\section{Imaging Studies and Repository}

The radiological imaging domain comprised the MRI CDE (Table 1). Hospital imaging protocols for 1.5-T T2weighted MRI scanners (General Electric) were used initially; after June 2016, patients mostly had a 3-T T2weighted MRI scan (Siemens). The axial T2-weighted sequence from each MRI examination was de-identified and DICOM files were converted to the 4D NifTI (.nii) file format using the MRIcron dcm2nii software package, freely available online (www.nitrc.org/projects/mricron). T2-hyperintense lesions were manually segmented using ITK-SNAP software (www.itksnap.org). ${ }^{35}$ Images were then registered to the Spinal Cord Toolbox for atlas-based, voxel-wise analyses. ${ }^{8}$ The MRI results were then coded according to the CDEs, and the BASIC scores were calculated..$^{31}$ All image processing and analyses were performed by a board-certified neuroradiologist (J.F.T.) blinded to patient demographic, socioeconomic, and clinical data.

\section{Outcome Measures}

The TRACK-SCI outcome battery consisted of core, supplemental, and emerging CDEs collected at 3, 6, and 12 months. All outcome measures, biospecimen extraction and processing, and examinations were conducted by trained personnel. For patients unable to answer on their own behalf, we included LAR responses.

At 3 months postinjury, patients were contacted by telephone to administer the following questionnaires: NINDS Rehabilitation Therapies, version 1.0; NINDS Injury Spinal Intervention and Spinal Procedures Basic Data Set, version 1.0; International Spinal Cord Injury (ISCI) Lower Urinary Tract Function Basic Data Set, version 1.0; ISCI Urinary Tract Infection Basic Data Set, version 1.0; ISCI Urodynamic Basic Data Set, version 1.0; ISCI Bowel Function Basic Data Set, version 1.1; ISCI Sexual Function Basic Data Set, version 1.0; ISCI Cardiovascular Basic Data Set, version 1.1; NINDS Upper Extremity Function (Fine Motor, ADL): Neuro-QOL Item Bank, version 1.0; ISCI Upper Extremity Basic Data Set, version 1.1; NINDS Lower Extremity Function (Mobility): Neuro-QOL Item Bank, version 1.0; NINDS Spinal Cord Independence Measure, version 1.0; ISCI Autonomic Dysfunction Following SCI;
ISCI Quality of Life Basic Data Set, version 1.0; NINDS Cognitive Function: Neuro-QOL Item Bank, version 2.0; NINDS Ability to Participate in Social Roles and Activities: Neuro-QOL Item Bank, version 1.0; NINDS Satisfaction with Social Roles and Activities: Neuro-QOL Item Bank, version 1.1; NINDS Anxiety: Neuro-QOL Item Bank, version 1.0; NINDS Depression: Neuro-QOL Item Bank, version 1.0; NINDS Emotional and Behavioral Dysfunction: Neuro-QOL Item Bank, version 1.0; NINDS Positive Affect and Well-Being: Neuro-QOL Item Bank, version 1.0; NINDS Fatigue: Neuro-QOL Item Bank, version 1.0; NINDS Stigma: Neuro-QOL Item Bank, version 1.0; Douleur Neuropathique 4; Location of Pain \& Pain Medication Questionnaire; and NINDS Pain Quality and Intensity Questionnaire (Table 1).

For the 6- and 12-month in-person assessments, patients underwent blood sample collection and ISNCSCI examination as well as the outcome battery administered at the 3-month follow-up. If a patient was unable to visit the research facilities for their 6- and/or 12-month assessments, questionnaires were administered via telephone; no blood samples or ISNCSCI examinations were collected or conducted for these cases.

\section{Statistical Analysis}

Descriptive statistics of patient demographics, injury characteristics, clinical interventions, and outcomes are presented. Excel (Microsoft Corp.) was used for all analyses.

\section{Results}

\section{Study Implementation and Enrollment}

The infrastructure for TRACK-SCI data collection was established within the first 18 months upon receipt of DOD funding. Within this period the following was achieved: TRACK-SCI electronic care report forms (eCRFs) were developed; IRB approval was obtained; personnel were recruited and hired; operational manuals for patient enrollment and follow-up assessments were created; and protocols for the collection, processing, and banking of biospecimens were designed and implemented.

In order to implement TRACK-SCI's mission, a leadership team of approximately 20 physicians, research faculty, data scientists, postdoctoral scholars, and research staff meet once every 2 weeks to discuss data, relevant study obstacles, and future directions. In addition, specialized "data meetings" are held once every 2 weeks for investigators specializing in data analysis and curation. Physicians from the departments of neurosurgery, neuroradiology, emergency medicine, orthopedic surgery, physical medicine and rehabilitation, anesthesiology, and neurology are present at these meetings. In addition, meetings are attended by appointed SCI champions in nursing, physical therapy, occupational therapy, and other departments.

Enrollment began in May 2015 with 160 SCI patients enrolled through July 1, 2019 (Fig. 2). Table 2 summarizes major demographic information and imaging characteristics of patients in the study. Of note, patients were not excluded if they had a history of substance abuse, mental health issues, or were homeless. Recruitment, enrollment, 


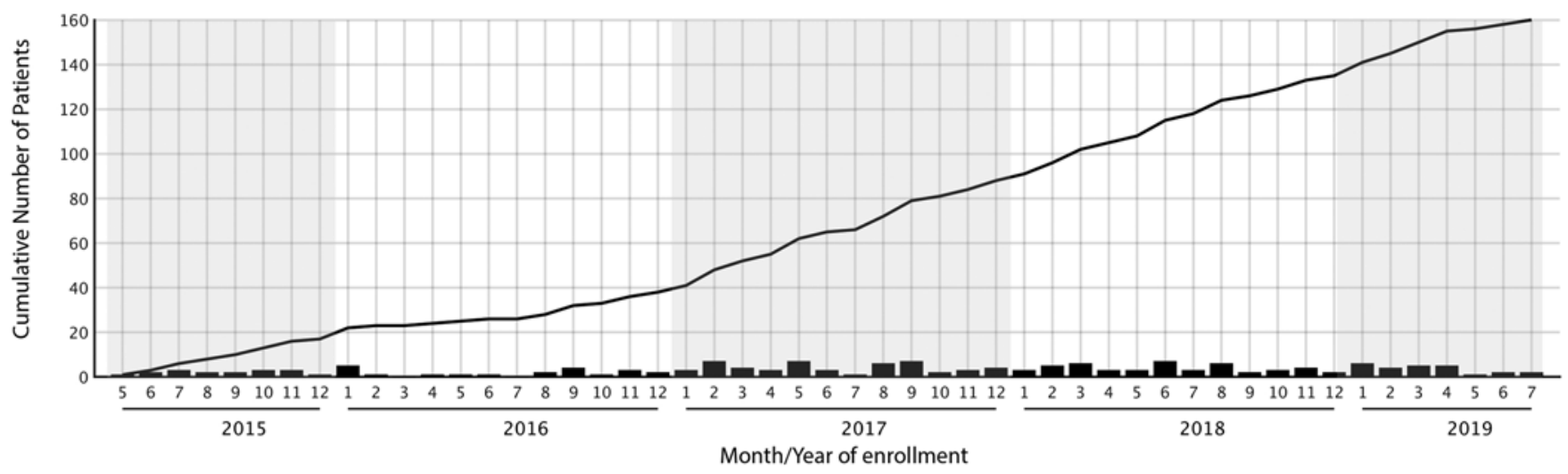

FIG. 2. Monthly (bars) and cumulative (line) enrollment rates for patients in the TRACK-SCI study.

and data collection were conducted by 1-2 full-time clinical research coordinators (CRCs) working 8 hours per day from 8 AM to 5 PM, 5 days per week. CRCs developed a call schedule to cover cases and enrollments on weekends and at night. To aid future investigators in budgeting for similar research, the workflow and estimated time on each task for patient enrollment and data collection are outlined in Fig. 3. How this time commitment translates to overall cost will depend on several factors. For example, if a particular center averages more than one SCI per week, then creating a prospective data registry will likely require at least two full-time CRCs. Depending on regional salary and institutional benefits requirements, financially supporting these positions will range from $\$ 100,000$ to $\$ 200,000$. Ultimately, the cost of the prospective registry would likely be much higher than this, especially if more granular data elements are collected.

\section{Demographic and Clinical Variables}

Of the 160 patients enrolled in the study, 115 (71.88\%) were male and $45(28.12 \%)$ were female. The overall age ranges and sex distributions within each decade are presented in Fig. 4. Males aged 18-29 and 50-59 years represented the most commonly injured patients in the study. The participant population was white $(43.8 \%)$, African American/black (15.0\%), Hispanic (12.5\%), Asian (18.1\%), Native Hawaiian/Pacific Islander (1.3\%), American Indian or Alaskan Native (1.3\%), and other or unknown (8.1\%).

\section{Injury Characteristics}

In terms of other injuries, $50 \%(n=80)$ of the patients had mild overall injuries (Injury Severity Score [ISS] $0-25), 24.3 \%(\mathrm{n}=39)$ had moderate injuries (ISS 26-50), and $4.38 \%(\mathrm{n}=7)$ had severe injuries (ISS 51-75); the remaining $21.3 \%(n=34)$ had no other injuries. Specifically for head injuries, $85.6 \%(n=137)$ had no evidence of head injury or a TBI Glasgow Coma Scale (GCS) score between 13 and 15 . For patients with more severe TBI, 5\% $(\mathrm{n}=8)$ had a presenting GCS score of 9-12, and $8.75 \%(n=14)$ had a presenting GCS score of 3-8 (data were unavailable for 1 patient). The most common injury etiology was a fall $(50 \%)$, followed by assault (15\%), motor vehicle collision $(12.5 \%)$, and other transportation-related injury including pedestrian or bicycle versus automobile (15\%; Table 2). On presentation, patients were most commonly categorized as AIS grade D (70 patients [43.8\%]), followed by AIS grade A (42 patients [26.3\%]), grade C (28 patients [17.5\%]), and grade $\mathrm{B}$ (5 patients [3.1\%]). Of note, for 15 patients, the presenting AIS grade could not be determined most commonly because patients could not be assessed at presentation given the injury severity, polytrauma, sedation, etc.

\section{Fracture Types and Interventions}

The majority of fracture cases occurred in the cervical spine $(62.5 \%)$, followed by the thoracic spine $(15 \%)$ and lumbar spine $(3.8 \%)$. For each patient, the fracture morphology was categorized using the $\mathrm{AO}$ spine classification system applied to thoracolumbar, subaxial cervical, and upper cervical fractures. ${ }^{14,29}$ We also categorized patients as having a central cord pattern of injury if MRI revealed significant cord injury and T2 signal in the absence of significant spinal fractures (Table 2). According to this system, patients most often presented with a central cord pattern of injury (70 patients [43.7\%]). Of the fracture types, AO type A patterns were the most common (21 patients [13.1\%]), followed by AO type B (20 patients [12.5\%]) and type $\mathrm{C}$ (17 patients [10.6\%]). We also used the BASIC score ${ }^{31}$ to measure the degree of intramedullary injury to the spine. These results suggest that there was an overall uniform distribution of injury types: BASIC scores 1 (15\%), 2 (13.8\%), 3 (8.8\%), and 4 (16.3\%). Of note, not all patients had a BASIC score calculated, mostly because they were taken to surgery before obtaining an MRI scan or because the MRI study was not interpretable due to motion artifact.

In terms of interventions, 122/160 patients (76.3\%) underwent surgical correction (Table 2). Most patients were treated with a posterior-only approach (93 patients [76.2\%]), followed by an anterior-only approach (15 patients [12.3\%]) and a combined posterior and anterior approach (14 patients [11.5\%]). In addition, 25 patients $(15.6 \%)$ also underwent postoperative CSF monitoring to measure spinal perfusion pressure through a lumbar drain.

\section{Follow-Up}

Because of the severe motor and sensory dysfunction 
TABLE 2. Demographic, clinical, imaging, and surgical information for $160 \mathrm{SCl}$ patients

\begin{tabular}{|c|c|c|}
\hline Characteristic & No. & $\%$ \\
\hline \multicolumn{3}{|l|}{ Sex } \\
\hline Female & 45 & 28.1 \\
\hline Male & 115 & 71.9 \\
\hline \multicolumn{3}{|l|}{ Injury mechanism } \\
\hline Assault & 24 & 15.0 \\
\hline Fall & 80 & 50.0 \\
\hline MVC & 20 & 12.5 \\
\hline Other transport-related injury & 24 & 15.0 \\
\hline Unknown & 20 & 12.5 \\
\hline \multicolumn{3}{|l|}{ Spinal level } \\
\hline Cervical & 100 & 62.5 \\
\hline Thoracic & 24 & 15.0 \\
\hline Lumbar & 6 & 3.8 \\
\hline Multiple levels & 30 & 18.8 \\
\hline \multicolumn{3}{|l|}{ Fracture type } \\
\hline Central cord & 70 & 43.7 \\
\hline AO type $A$ & 21 & 13.1 \\
\hline AO type B & 20 & 12.5 \\
\hline AO type $\mathrm{C}$ & 17 & 10.6 \\
\hline Other & 32 & 20.0 \\
\hline \multicolumn{3}{|l|}{ MRI characteristic } \\
\hline BASIC score 1 & 24 & 15.0 \\
\hline BASIC score 2 & 22 & 13.8 \\
\hline BASIC score 3 & 14 & 8.8 \\
\hline BASIC score 4 & 26 & 16.3 \\
\hline Unable to detect & 74 & 46.3 \\
\hline \multicolumn{3}{|l|}{ Presentation AIS grade } \\
\hline A & 42 & 26.3 \\
\hline $\mathrm{B}$ & 5 & 3.1 \\
\hline C & 28 & 17.5 \\
\hline$D$ & 70 & 43.8 \\
\hline Unable to detect & 15 & 9.4 \\
\hline Surgery & 122 & 76.3 \\
\hline Ant only & 15 & 12.3 \\
\hline Pst only & 93 & 76.2 \\
\hline Ant \& pst & 14 & 11.5 \\
\hline \multicolumn{3}{|l|}{ Lumbar drain } \\
\hline Yes & 25 & 15.6 \\
\hline No & 135 & 84.4 \\
\hline
\end{tabular}

Ant = anterior; $\mathrm{MVC}=$ motor vehicle collision; pst = posterior.

and additional comorbidities associated with SCIs, as well as our broad inclusion criteria, follow-up assessments were difficult to obtain. For the 3-, 6-, and 12-month follow-ups, $45.6 \%, 45 \%$, and $36.3 \%$ of patients, respectively, completed their assessments. In-person visits for the 6- and 12-month follow-ups were completed for $16.3 \%$ and $11.9 \%$ of patients, respectively (Table 3 ). For patients who could not return to the research facility, phone assessments were conducted. Follow-up assessments were missed because the patients could not be contacted, withdrew from the study, or were deceased.

\section{Discussion}

The TRACK-SCI prospective database was designed using the principles of CDEs with the goal of standardizing SCI outcomes research across an ultimately multiinstitutional effort with up to 12 months of follow-up data. In forming this registry, we have partnered with several other centers including UCSF-Fresno and The Ohio State University medical center. We believe that it is critically important to integrate data across institutions for several reasons. First, increasing the number of centers will increase the overall number of patients in the registry. Second, by partnering with centers outside of academia, it will be possible to use the resources available at a major academic center to help create an SCI data registry at sites that may not have the grant or financial support to do so. Third, the addition of several centers will increase the diversity of the patient population, helping to translate the results of the study to a more general population.

Outcome measurement is becoming critical in the new paradigm of value-based care. ${ }^{27}$ Being able to accurately report outcomes is another benefit of clinical registries. ${ }^{26}$ As the Centers for Medicare \& Medicaid Services (CMS) institutes alternative payment models, it is important to have registries that will meet their Qualified Clinical Data Registry requirements. Subspecialty registries are one of the ways that physicians can report outcomes to the CMS. The field of neurosurgery has been a leader in this effort with the Quality Outcomes Database (QOD; formerly the National Neurosurgery Quality and Outcomes Database [ $\left.\mathrm{N}^{2} \mathrm{QOD}\right]$ ) recently receiving CMS approval as a physician quality reporting system. ${ }^{26}$ We expect that TRACK-SCI will also eventually be useful in that regard.

Aside from outcome measurement, the database and the highly granular CDEs will be useful to prospectively investigate how different clinical treatments correlate with outcome after SCI. Already, the pilot data and early prospective results from TRACK-SCI have contributed to a number of advances, including blood pressure management, ${ }^{6,11,15}$ radiographic evaluation of SCI, ${ }^{16,31}$ surgical timing, ${ }^{5}$ motor evoked potentials in the assessment of injury severity, ${ }^{10}$ complication avoidance,,${ }^{12,28}$ and the role of spinal perfusion pressure. ${ }^{32,33}$ Moving forward, we expect the power of these investigations to increase as the numbers of patients and clinical sites increase.

We also included the collection of biospecimens using the protocols established in the TRACK-TBI study. These protocols were used to collect, process, and store blood samples for proteomic and genetic analyses beginning in May 2016. The goal of the biospecimen project is to discover blood biomarkers to help in early detection and assessment of SCI and/or predict long-term neurological recovery. Analysis of the results of this effort and the development of potential predictive models relating early critical care to outcomes are in process.

Regarding limitations of the study, the low rate of pediatric SCIs prevented pediatric patient enrollment. Also, 


\begin{tabular}{|l|c|}
\hline Patient Enrollment & $\begin{array}{l}\text { Time } \\
\text { (mins) }\end{array}$ \\
\hline Identify Exclusions & 15 \\
\hline Verify CT/MRI performed & 5 \\
\hline Gather documents/supplies & 10 \\
\hline Explain study for consent & 30 \\
\hline Answer questions & 15 \\
\hline Patientfamily decision time & 30 \\
\hline Signing consent forms & 5 \\
\hline Total & $\mathbf{1 1 0}$ \\
\hline
\end{tabular}

\begin{tabular}{|l|c|}
\hline \multicolumn{1}{|c|}{ Neuroimaging' } & $\begin{array}{l}\text { Time } \\
\text { (mins) }\end{array}$ \\
\hline Radiologist read and dictation & 30 \\
\hline Abstract results for CRF & 5 \\
\hline Enter results into database & 10 \\
\hline Total & $\mathbf{4 5}$ \\
\hline $\begin{array}{l}{ }^{1} \text { All scans were conducted as standard of } \\
\text { care }\end{array}$ \\
\hline
\end{tabular}

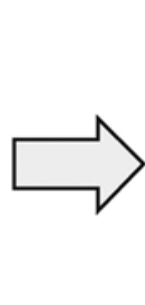

\begin{tabular}{|l|c|}
\hline Outcome at $\mathbf{3}$ months & $\begin{array}{l}\text { Time } \\
\text { (mins) }\end{array}$ \\
\hline Contact patient to schedule call & 30 \\
\hline Administer questionnaires & 90 \\
\hline Process patient reimbursements & 15 \\
\hline Enter results into database & 45 \\
\hline Total & $\mathbf{1 8 0}$ \\
\hline
\end{tabular}

\begin{tabular}{|l|c|}
\hline $\begin{array}{l}\text { Demographics and } \\
\text { Clinical }\end{array}$ & $\begin{array}{l}\text { Time } \\
\text { (mins) }\end{array}$ \\
\hline Contact information & 5 \\
\hline Patient demographics & 15 \\
\hline Health history & 20 \\
\hline Injury-related events & 30 \\
\hline Assessment and examinations & 60 \\
\hline Enter CRF into REDCap & 50 \\
\hline Total & $\mathbf{1 8 0}$ \\
\hline
\end{tabular}

\begin{tabular}{|l|c|}
\hline $\begin{array}{l}\text { Biospecimen Collection \& } \\
\text { Processing }\end{array}$ & $\begin{array}{l}\text { Time } \\
\text { (mins) }\end{array}$ \\
\hline Acute 24-hour blood draw & 5 \\
\hline Centrifuge, aliquot \& freeze & 60 \\
\hline Log vials and processing time & 10 \\
\hline $48^{-+}+72^{-+}+120$-hour blood draw & 15 \\
\hline $48-+72^{-+}+120$-hour Centrifuge, aliquot \& freeze & 180 \\
\hline $48-+72^{-+} 120$-hour Log vials and processing time & 60 \\
\hline $\begin{array}{l}\text { Total 24 hour } \\
\text { Total 24-120 hour }\end{array}$ & $\begin{array}{c}\mathbf{7 5} \\
\mathbf{3 0 0}\end{array}$ \\
\hline
\end{tabular}
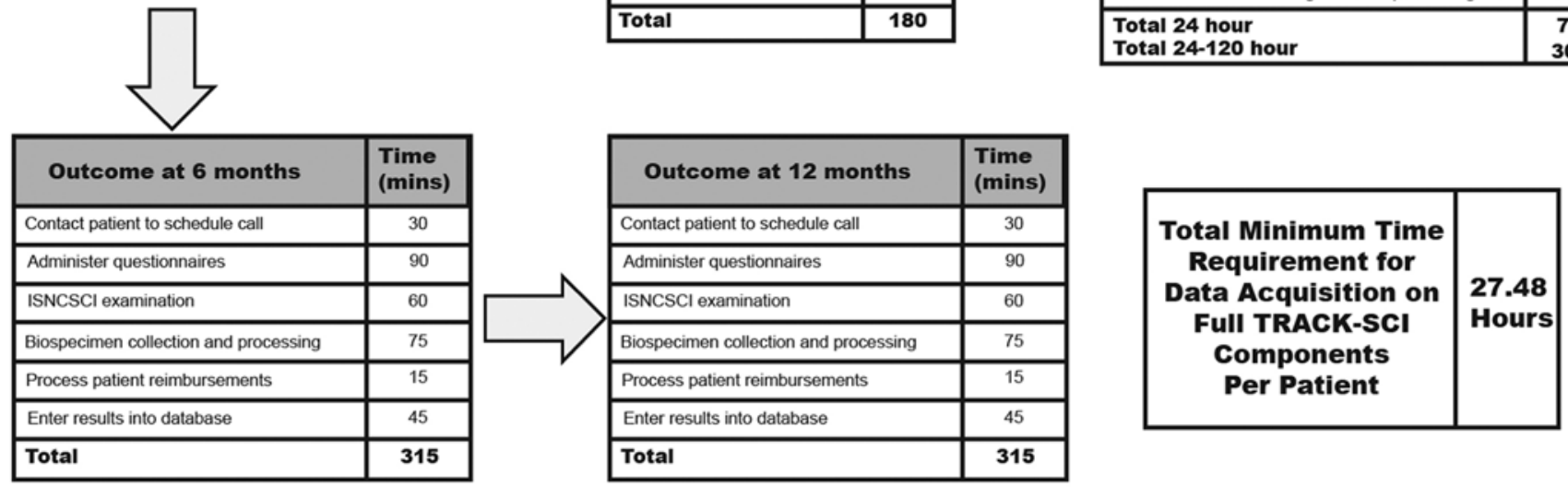

FIG. 3. Workflow diagram of tasks needed to complete enrollment by full-time TRACK-SCI CRCs. The time of each task is given in minutes (mins), and the total time in hours for enrollment of one patient is given in the lower right box. CRF = care report form.

the lack of validated SCI CDE outcome measures for non-English-speaking participants limited our ability to fully capture accurate quality of life data at the follow-up time points. In addition, a low follow-up rate is a com-

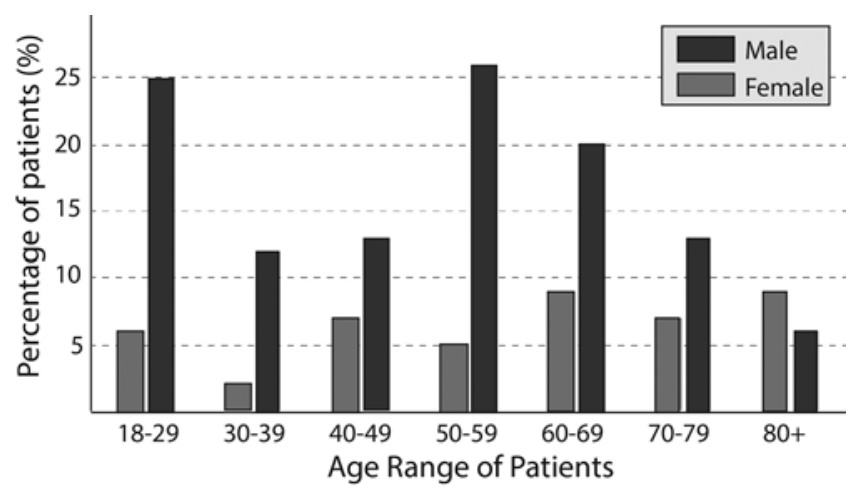

FIG. 4. Overall age (in years) and sex distributions of patients enrolled in the TRACK-SCI study. mon issue in SCI research and is more pronounced at our institution, which is a county hospital for a large metropolitan area. There are a number of strategies that could be implemented to address this issue. First, the inclusion of several centers (as done here) pools data over a larger area and increases the number of follow-up data points. Second, linking electronic medical records across several institutions will allow patients to be tracked and monitored more easily at discharge. Third, the concept of a prospective registry itself can be modified: by limiting data points to medical records of future health encounters, it would be possible to create an extended quality improvement database, which would provide a modest measure of outcome after SCI with lower costs and less institutional support than a prospective registry.

Additionally, ISNCSCI assessments were performed during admission, in hospital, and at the 6- and 12-month follow-ups. These examinations are considered the gold standard for neurological assessment after SCI. ${ }^{19}$ However, their implementation can be challenging in an ultra-acute trauma setting, especially if patients require immediate 
TABLE 3. TRACK-SCI follow-up assessment completion rates

\begin{tabular}{lccc}
\hline & \multicolumn{3}{c}{ FU Assessment } \\
\cline { 2 - 4 } & 3 Mos $^{*}$ & 6 Mos† & 12 Mos $\ddagger$ \\
\hline Total assessments completed & $73(45.6 \%)$ & $72(45 \%)$ & $58(36.3 \%)$ \\
\hline Via phone & 73 & 46 & 39 \\
\hline In person & NA & 26 & 19 \\
\hline Assessment missed & $55(34.4 \%)$ & $52(32.5 \%)$ & $41(25.6 \%)$ \\
\hline Patient withdrew from study & $18(11.3 \%)$ & $21(13.1 \%)$ & $25(15.6 \%)$ \\
\hline Patient died & $13(8.1 \%)$ & $13(8.1 \%)$ & $14(8.8 \%)$ \\
\hline NA = not applicable. & & & \\
* One patient was awaiting their 3-month phone appointment. \\
$\begin{array}{l}\dagger \text { Two patients were awaiting their 6-month appointment. } \\
\text { † Twenty-two patients were awaiting their 12-month appointment. }\end{array}$
\end{tabular}

surgical stabilization. In addition, this examination is difficult to perform in patients with an altered mental state or ongoing sedation. ISNCSCI examinations at the 6- and 12-month follow-ups were easier to perform but were limited by low in-person follow-up rates, which were $16 \%$ and $11 \%$ at 6 and 12 months, respectively. We believe the low rate of follow-up is reflective of real-world patients in a major metropolitan area and highlights the need for shortened ISNCSCI examinations to maximize the amount of data in these situations. Future research should validate shortened versions of the ISNCSCI assessment to expedite reliable clinical evaluation.

\section{Conclusions}

We have established the feasibility of SCI CDE implementation in a pilot, multicenter, prospective observational study. Through multidisciplinary collaboration, integrated protocols and procedures were developed for collecting standardized high-quality data. Opportunities for improvement in data procurement and follow-up were identified. Through the use of standardized SCI CDEs and the expansion of additional future institutional collaborations, we hope to advance SCI research.

\section{Acknowledgments}

The current work was supported by Department of Defense grant nos. W81XWH-13-1-0297 (Effects of Early Acute Care on Autonomic Outcomes in SCI: Bedside to Bench and Back) and W81XWH-16-1-0497 (Early Critical Care Decisions and Outcomes after SCI: TRACK-SCI), the Craig H. Neilsen grant Center of Excellence in Spinal Cord Injury: TRACK-SCI, and the Wings for Life grant no. WFL-US-07-18 (Discovering Blood RNA Biomarkers for Diagnosis of SCI Severity and/or Prognosis of Neurological Recovery).

\section{References}

1. Baldi I, Engelhardt J, Bonnet C, Bauchet L, Berteaud E, Grüber A, et al: Epidemiology of meningiomas. Neurochirurgie 64:5-14, 2018

2. Biering-Sørensen F, Alai S, Anderson K, Charlifue S, Chen Y, DeVivo M, et al: Common data elements for spinal cord injury clinical research: a National Institute for Neurological Disorders and Stroke project. Spinal Cord 53:265-277, 2015
3. Biering-Sørensen F, Charlifue S, DeVivo M, Noonan V, Post $\mathrm{M}$, Stripling T, et al: International spinal cord injury data sets. Spinal Cord 44:530-534, 2006

4. Biering-Sørensen F, Noonan VK: Standardization of data for clinical use and research in spinal cord injury. Brain Sci 6:29, 2016

5. Burke JF, Yue JK, Ngwenya LB, Winkler EA, Talbott JF, Pan JZ, et al: Ultra-early ( $<12$ hours) surgery correlates with higher rate of American Spinal Injury Association Impairment Scale conversion after cervical spinal cord injury. Neurosurgery 85:199-203, 2019

6. Catapano JS, John Hawryluk GW, Whetstone W, Saigal R, Ferguson A, Talbott J, et al: Higher mean arterial pressure values correlate with neurologic improvement in patients with initially complete spinal cord injuries. World Neurosurg 96:72-79, 2016

7. Curt A, Schwab ME, Dietz V: Providing the clinical basis for new interventional therapies: refined diagnosis and assessment of recovery after spinal cord injury. Spinal Cord 42:1-6, 2004

8. De Leener B, Lévy S, Dupont SM, Fonov VS, Stikov N, Louis Collins D, et al: SCT: Spinal Cord Toolbox, an open-source software for processing spinal cord MRI data. Neuroimage 145 (Pt A):24-43, 2017

9. DeVivo M, Chen Y, Mennemeyer S, Deutsch A: Costs of care following spinal cord injury. Top Spinal Cord Inj Rehabil 16:1-9, 2011

10. Dhall SS, Haefeli J, Talbott JF, Ferguson AR, Readdy WJ, Bresnahan JC, et al: Motor evoked potentials correlate with magnetic resonance imaging and early recovery after acute spinal cord injury. Neurosurgery 82:870-876, 2018

11. DiGiorgio A, Burke J, Winkler E, Talbott J, Ferguson A, Beattie M, et al: Aggressive surgical treatment and critical care are associated with improved neurologic recovery in complete SCI. J Neurosurg 128:54-55, 2018 (Abstract)

12. DiGiorgio AM, Tsolinas R, Alazzeh M, Haefeli J, Talbott JF, Ferguson AR, et al: Safety and effectiveness of early chemical deep venous thrombosis prophylaxis after spinal cord injury: pilot prospective data. Neurosurg Focus 43(5):E21, 2017

13. Fehlings MG, Nakashima H, Nagoshi N, Chow DS, Grossman RG, Kopjar B: Rationale, design and critical end points for the Riluzole in Acute Spinal Cord Injury Study (RISCIS): a randomized, double-blinded, placebo-controlled parallel multi-center trial. Spinal Cord 54:8-15, 2016

14. Fehlings MG, Smith JS, Kopjar B, Arnold PM, Yoon ST, Vaccaro AR, et al: Perioperative and delayed complications associated with the surgical treatment of cervical spondylotic myelopathy based on 302 patients from the AOSpine North America Cervical Spondylotic Myelopathy Study. J Neurosurg Spine 16:425-432, 2012

15. Hawryluk G, Whetstone W, Saigal R, Ferguson A, Talbott J, Bresnahan J, et al: Mean arterial blood pressure correlates with neurological recovery after human spinal cord injury: analysis of high frequency physiologic data. J Neurotrauma 32:1958-1967, 2015

16. Huie JR, Talbott J, Singh V, Fernandez XD, Tsolinas R, Pascual L, et al: Data-driven comparison of acute imaging biomarkers for spinal cord injury: a prospective TRACK-SCI pilot study (S42.006). Neurology 90 (15 Suppl):S42.006, 2018

17. Jung KW, Park KH, Ha J, Lee SH, Won YJ, Yoo H: Incidence of primary spinal cord, spinal meninges, and cauda equina tumors in Korea, 2006-2010. Cancer Res Treat 47:166-172, 2015

18. Kim T, Kwon OK, Bang JS, Lee H, Kim JE, Kang HS, et al: Epidemiology of ruptured brain arteriovenous malformation: a National Cohort Study in Korea. J Neurosurg 130:19651970,2019 
19. Kirshblum SC, Burns SP, Biering-Sorensen F, Donovan W, Graves DE, Jha A, et al: International standards for neurological classification of spinal cord injury (revised 2011). J Spinal Cord Med 34:535-546, 2011

20. Laws ER, Parney IF, Huang W, Anderson F, Morris AM, Asher A, et al: Survival following surgery and prognostic factors for recently diagnosed malignant glioma: data from the Glioma Outcomes Project. J Neurosurg 99:467-473, 2003

21. McDougall CG, Spetzler RF, Zabramski JM, Partovi S, Hills NK, Nakaji P, et al: The Barrow Ruptured Aneurysm Trial. J Neurosurg 116:135-144, 2012

22. National Spinal Cord Injury Statistical Center: Spinal Cord Injury Facts and Figures at a Glance. Birmingham, AL: University of Alabama at Birmingham, 2016

23. Noonan V: Working Towards an International Spinal Cord Injury Data Set. Summary of Workshop Proceedings. Vancounver, 2002

24. Noonan VK, Kwon BK, Soril L, Fehlings MG, Hurlbert RJ, Townson A, et al: The Rick Hansen Spinal Cord Injury Registry (RHSCIR): a national patient-registry. Spinal Cord 50:22-27, 2012

25. Ostrom QT, Gittleman H, Fulop J, Liu M, Blanda R, Kromer $\mathrm{C}$, et al: CBTRUS statistical report: primary brain and central nervous system tumors diagnosed in the United States in 2008-2012. Neuro Oncol 17 (Suppl 4):iv1-iv62, 2015

26. Parker SL, McGirt MJ, Bekelis K, Holland CM, Davies J, Devin CJ, et al: The National Neurosurgery Quality and Outcomes Database Qualified Clinical Data Registry: 2015 measure specifications and rationale. Neurosurg Focus 39(6):E4, 2015

27. Porter ME: What is value in health care? N Engl J Med 363:2477-2481, 2010

28. Readdy WJ, Whetstone WD, Ferguson AR, Talbott JF, Inoue $\mathrm{T}$, Saigal R, et al: Complications and outcomes of vasopressor usage in acute traumatic central cord syndrome. J Neurosurg Spine 23:574-580, 2015

29. Reinhold M, Audigé L, Schnake KJ, Bellabarba C, Dai LY, Oner FC: AO Spine Injury Classification System: a revision proposal for the thoracic and lumbar spine. Eur Spine J 22:2184-2201, 2013

30. Sebastian AS: Database research in spine surgery. Clin Spine Surg 29:427-429, 2016

31. Talbott JF, Whetstone WD, Readdy WJ, Ferguson AR, Bresnahan JC, Saigal R, et al: The Brain and Spinal Injury Center score: a novel, simple, and reproducible method for assessing the severity of acute cervical spinal cord injury with axial T2-weighted MRI findings. J Neurosurg Spine 23:495-504, 2015

32. Yue JK, Hemmerle DD, Winkler EA, Thomas LH, Fernandez $\mathrm{XD}$, Kyritsis N, et al: Clinical implementation of novel spinal cord perfusion pressure protocol in acute traumatic spinal cord injury at U.S. level I trauma center: TRACK-SCI study. World Neurosurg 133:e391-e396, 2019
33. Yue JK, Hemmerle DP, Deng H, Winkler EA, Thomas LH, Fernandez XD, et al: Initial experience of spinal cord perfusion pressure goals in lieu of mean arterial pressure goals in acute traumatic spinal cord injury at a United States level I trauma center: a transforming research and clinical knowledge-spinal cord injury study. Neurosurgery 66 (Suppl 1):nyz310-444, 2019

34. Yue JK, Vassar MJ, Lingsma HF, Cooper SR, Okonkwo DO, Valadka AB, et al: Transforming research and clinical knowledge in traumatic brain injury pilot: multicenter implementation of the common data elements for traumatic brain injury. J Neurotrauma 30:1831-1844, 2013

35. Yushkevich PA, Piven J, Hazlett HC, Smith RG, Ho S, Gee JC, et al: User-guided 3D active contour segmentation of anatomical structures: significantly improved efficiency and reliability. Neuroimage 31:1116-1128, 2006

\section{Disclosures}

The authors report no conflict of interest concerning the materials and methods used in this study or the findings specified in this paper.

\section{Author Contributions}

Conception and design: Ferguson, Manley, Bresnahan, Beattie, Dhall. Acquisition of data: Thomas, Duong-Fernandez, Harris, Manley, Bresnahan, Beattie, Dhall. Analysis and interpretation of data: Burke, Tsolinas, DiGiorgio, Thomas, Duong-Fernandez, Harris, Yue, Winkler, Suen, Pascual, Ferguson, Huie, Pan, Hemmerle, Singh, Torres-Espin, Omondi, Kyritsis, Haefeli, Weinstein, Kuo, Taggard, Talbott, Whetstone, Manley, Bresnahan, Beattie, Dhall. Drafting the article: all authors. Critically revising the article: all authors. Reviewed submitted version of manuscript: all authors. Approved the final version of the manuscript on behalf of all authors: Burke. Statistical analysis: Burke, Tsolinas, DiGiorgio, Yue, Winkler, Pascual, Ferguson, Huie, Pan, Hemmerle, Singh, Torres-Espin, Omondi, Kyritsis, Talbott, Whetstone, Manley, Bresnahan, Beattie, Dhall. Administrative/ technical/material support: Burke, Tsolinas, DiGiorgio, Thomas, Duong-Fernandez, Harris, Yue, Suen, Pascual, Ferguson, Huie, Pan, Hemmerle, Singh, Torres-Espin, Omondi, Kyritsis, Kuo, Taggard, Talbott, Whetstone, Manley, Bresnahan, Beattie, Dhall. Study supervision: Ferguson, Manley, Bresnahan, Beattie, Dhall.

\section{Correspondence}

John F. Burke: University of California, San Francisco, CA. john. burke@ucsf.edu. 